\title{
Modélisation de la formation et de l'évolution non linéaire des barres en croissant de la côte aquitaine.
}

\author{
Roland Garnier ${ }^{1,2}$, rgarnier@fa.upc.edu. \\ Philippe Bonneton ${ }^{2}$, p.bonneton@epoc.u-bordeaux1.fr. \\ Albert Falqués ${ }^{1}$, falques@fa.upc.edu. \\ Daniel Calvete ${ }^{1}$, calvete@fa.upc.edu. \\ ${ }^{1}$ Universitat Politècnica de Catalunya, Departament de Física \\ Aplicada, 08034 Barcelone, Espagne. \\ ${ }^{2}$ Université de Bordeaux I, DGO-UMR 5805 avenue des Facultés, \\ 33405 Talence cedex, France.
}

\section{$\underline{\text { Résumé }}$}

Les barres en croissant émergent à partir d'instabilités internes du système morphodynamique par un processus d'auto-organisation couplant la topographie et l'hydrodynamique. Leur évolution à long terme sera étudiée dans le cas réel des plages d'Aquitaine à l'aide du modèle numérique 2DH : MORFO55. Pour la première fois, l'état d'équilibre d'un système de barres en croissant est obtenu, lorsque la condition de houle est stationnaire. Dû aux interactions non linéaires, la longueur d'onde finale du système n'augmente pas forcément avec la taille des vagues. Enfin, l'étude de conditions de houle variable, suggère que l'état d'équilibre peut dépendre des conditions initiales.

\begin{abstract}
Crescentic bars emerge as free instabilities of the coupling between topography and water motion. Their long term behaviour will be studied in the real case of the French Aquitaine beaches by using the 2DH numerical model MORFO55. For the first time, the equilibrium state of a crescentic bar system is obtained, in the case of steady incident wave conditions. Due to non linear interactions, the final wave length of the system does not inevitably increase with the incident wave height. Finally, the study of variable incident wave conditions suggests that the equilibrium state may depend on the initial conditions.
\end{abstract}

\section{Mots clés}

morphodynamique, modélisation non linéaire, auto-organisation, instabliltés, barres en croissant 


\section{Introduction}

Les barres en croissant apparaissent de manière persistante dans la zone subtidale de la plupart des côtes sableuses exposées à la houle telle que la côte aquitaine ${ }^{3 ; 7}$. Ces structures rythmiques se développent généralement sur une barre longitudinale. Elles sont caractérisées par une longueur d'onde qui dépendrait de la position transversale de la barre longitudinale ${ }^{4}$. Or, sur la côte aquitaine où leur longueur d'onde peut varier de $300 \mathrm{~m}$ à $1500 \mathrm{~m}$, le faible mouvement transversal de la barre longitudinale suggère que d'autres variables influent sur la longueur d'onde.

Les études actuelles démontrent qu'elles émergent à partir d'instabilités internes du système morphodynamique par un processus d'auto-organisation couplant la topographie et l'hydrodynamique ${ }^{5}$. En particulier, elles ont fait l'objet de nombreuses études numériques utilisant des modèles linéaires ${ }^{2}$ ou non linéaires ${ }^{1 ; 4 ; 3}$. Cependant ces derniers modèles décrivent uniquement la formation des barres ou les étapes initiales de leur évolution.

L'intérêt principal de cette contribution sera de modéliser le comportement à long terme des barres en croissant des plages d'Aquitaine et d'obtenir un état d'équilibre pour une condition de houle stationnaire. Il sera aussi abordé l'effet d'une variation des conditions de houle. Le modèle numérique MORFO55 utilisé dans le cas d'une plage plane idéalisée pour analyser l'évolution non linéaire des barres obliques et transversales proches de la ligne de côte permettant, en particulier, d'étudier le mécanisme de saturation ${ }^{6}$ sera appliqué au cas réel des plages d'Aquitaine.

\section{Le modèle MORFO55}

Le modèle MORFO55 est un modèle numérique non linéaire qui résout les équations de Navier-Stokes en eau peu profonde, moyennées sur une colonne d'eau et sur une période de vague, couplées avec l'équation de conservation du sédiment. La ligne de côte, définie par $x_{1}=0 \mathrm{~m}$, est supposée rectiligne. L'axe $x_{1}$ représente l'axe transversal et l'axe $x_{2}$, l'axe longitudinal.

\subsection{Hydrodynamique}

L'hydrodynamique est gouvernée par les équations de conservation de la masse (1) et de la quantité de mouvement (2) décrites par $\mathrm{Mei}^{8}$ :

$$
\frac{\partial D}{\partial t}+\frac{\partial}{\partial x_{j}}\left(D v_{j}\right)=0,
$$




$$
\frac{\partial v_{i}}{\partial t}+v_{j} \frac{\partial v_{i}}{\partial x_{j}}=-g \frac{\partial z_{s}}{\partial x_{i}}-\frac{1}{\rho D} \frac{\partial}{\partial x_{j}}\left(S_{i j}^{\prime}-S_{i j}^{\prime \prime}\right)-\frac{\tau_{b i}}{\rho D}, \quad i=1,2,
$$

où $D\left(x_{1}, x_{2}, t\right)$ est la profondeur d'eau moyenne, $\vec{v}\left(x_{1}, x_{2}, t\right)$ est le vecteur de courant moyen $\left(\vec{v}=\left(v_{1}, v_{2}\right)\right), z_{s}\left(x_{1}, x_{2}, t\right)$ est le niveau d'eau moyen, $g$ est l'accélération de la pesanteur, $\rho$ est la densité de l'eau, $\bar{S}^{\prime}$ est le tenseur de radiation, $\overline{\bar{S}^{\prime}}$ est le tenseur de turbulence de Reynolds et $\vec{\tau}_{b}$ est le vecteur des contraintes au fond. La transformation des vagues est régie de la manière suivante. Premièrement, nous utilisons l'équation de densité d'énergie (3) qui inclue l'interaction vagues-courant ainsi que les vagues irrégulières :

$$
\frac{\partial E}{\partial t}+\frac{\partial}{\partial x_{j}}\left(\left(v_{j}+c_{g j}\right) E\right)+S_{i j}^{\prime} \frac{\partial v_{j}}{\partial x_{i}}=-\varepsilon,
$$

où $E\left(x_{1}, x_{2}, t\right)$ est la densité d'énergie définie par $E=\frac{1}{8} \rho g H_{\mathrm{rms}}^{2}, H_{r m s}$ est la hauteur rms de vague, $\overrightarrow{c_{g}}$ est la vitesse de groupe des vagues et $\varepsilon$ est le taux de dissipation dû au déferlement et à la friction du fond. Deuxièmement, le vecteur d'onde $\vec{k}\left(x_{1}, x_{2}, t\right)$ satisfait la relation de dispersion (4) et la loi de Snell-Descartes (5) :

$$
\begin{gathered}
\sigma^{2}=g k \tanh k D, \\
k \sin \theta=k_{\infty} \sin \theta_{\infty},
\end{gathered}
$$

où $k$ est le module de $\vec{k}$ et $\theta$ l'angle entre les fronts d'ondes et l'axe $x_{1}$. La fréquence angulaire $\sigma=2 \pi / T$ est supposée constante, $T$ étant la période des vagues.

\subsection{Morphodynamique}

L'évolution du niveau topographique $z_{b}\left(x_{1}, x_{2}, t\right)$ couplé à $\mathrm{D}$ par la relation $D=$ $z_{s}-z_{b}$ est régie par l'équation de conservation du sédiment (6) :

$$
\frac{\partial z_{b}}{\partial t}+\frac{1}{1-p} \frac{\partial q_{j}}{\partial x_{j}}=0
$$

où $\vec{q}$ est le vecteur de flux sédimentaire horizontal et $p$ la porosité du sable. La loi de transport de sédiment (7) s'écrit :

$$
\vec{q}=\alpha\left(\vec{v}-\gamma u_{r m s}^{b} \vec{\nabla} h\right),
$$

où $h=z_{b}-z_{b}^{0}$ est la perturbation du fond par rapport à la topographie d'équilibre, $u_{r m s}^{b}$ est le module de la vitesse orbitale moyenne des vagues, $\gamma$ est le paramètre de transport dû à la pente du fond et $\alpha$ est le facteur de mélange (stirring) obtenu d'après la formule de Soulsby et Van Rijn ${ }^{9}$.

\subsection{Méthodes numériques}


Les cinq équations (1), (2) $)_{i=1,2}$, (3) et (6) avec leur cinq inconnues $z_{s}\left(x_{1}, x_{2}, t\right)$, $v_{1}\left(x_{1}, x_{2}, t\right), v_{2}\left(x_{1}, x_{2}, t\right), H_{r m s}\left(x_{1}, x_{2}, t\right)$ et $z_{b}\left(x_{1}, x_{2}, t\right)$ ont été résolues numériquement afin de calculer l'évolution temporelle de la plage en équilibre perturbé. Un schéma numérique en différences finies est utilisé ${ }^{1}$, avec pour les dérivés temporelles, un schéma explicite du type Adams-Bashforth, et pour les dérivés spatiales, un schéma centré retardé.

\subsection{Conditions limites}

Au niveau des frontières latérales, chaque variable et sa dérivé première sont supposées périodiques. Afin de s'assurer que ces conditions périodiques n'aient pas d'incidence sur les longueurs d'onde des structures formées, des experiences ont été réalisées en variant la largeur du domaine. Au large, $v_{1}$ et $v_{2}$ diminuent exponentiellement, $H_{r m s}$ est imposée, $z_{s}$ et $z_{b}$ respectent leurs équations respectives. Sur la ligne de côte, un mur absorbant d'une hauteur de $10 \mathrm{~cm}$ impose une vitesse nulle.

\subsection{Bathymétrie d'équilibre}

La bathymétrie d'équilibre, longitudinalement uniforme, a été calculée ${ }^{3}$ à partir d'une série de profils bathymétriques relevés à la plage du Truc Vert à niveau d'eau moyen $\left(z_{b}^{0}\left(x_{1}\right)\right)$. En utilisant cette bathymétrie initiale, le système bathymétrique reste stable car le transport transversal, i.e. le transport dû au courant de retour et à l'asymétrie des vagues, est négligé. Dans le but d'exciter les instabilités, une perturbation aléatoire d'une amplitude de $10 \mathrm{~cm}\left(h\left(x_{1}, x_{2}, t=0\right)=h_{0}\left(x_{1}, x_{2}\right)\right)$ est ajoutée à cette bathymétrie d'équilibre telle que la bathymétrie initiale (8) devient :

$$
z_{b}\left(x_{1}, x_{2}, t=0\right)=z_{b}^{0}\left(x_{1}\right)+h_{0}\left(x_{1}, x_{2}\right) .
$$

\subsection{Expériences}

Dans la suite de cette contribution, les axes $x_{1}$ et $x_{2}$ seront notés axes $x$ et $y$. De même, les composantes du vecteur vitesse $v_{1}$ et $v_{2}$ deviennent $u$ et $v$. Le domaine de calcul est une grille rectangulaire délimitée par $0 \leq x \leq L_{x}=1000 \mathrm{~m}$ dans la direction transversale et $0 \leq y \leq L_{y}=9000 \mathrm{~m}$ dans la direction longitudinale. La ligne de côte est définie par $x=0 \mathrm{~m}$.

Le pas de temps est de $18 \mathrm{~s}$ et le pas d'espace est de $25 * 25 \mathrm{~m}^{2}$. Deux conditions de houle seront étudiées : une houle frontale, de période $T=12 \mathrm{~s}$, de hauteur au large (à $x=L_{x}$ ) (a) $H_{r m s}=1.5 \mathrm{~m}$ et (b) $H_{r m s}=1.8 \mathrm{~m}$.

\section{Résultats}




\section{1. États basiques}
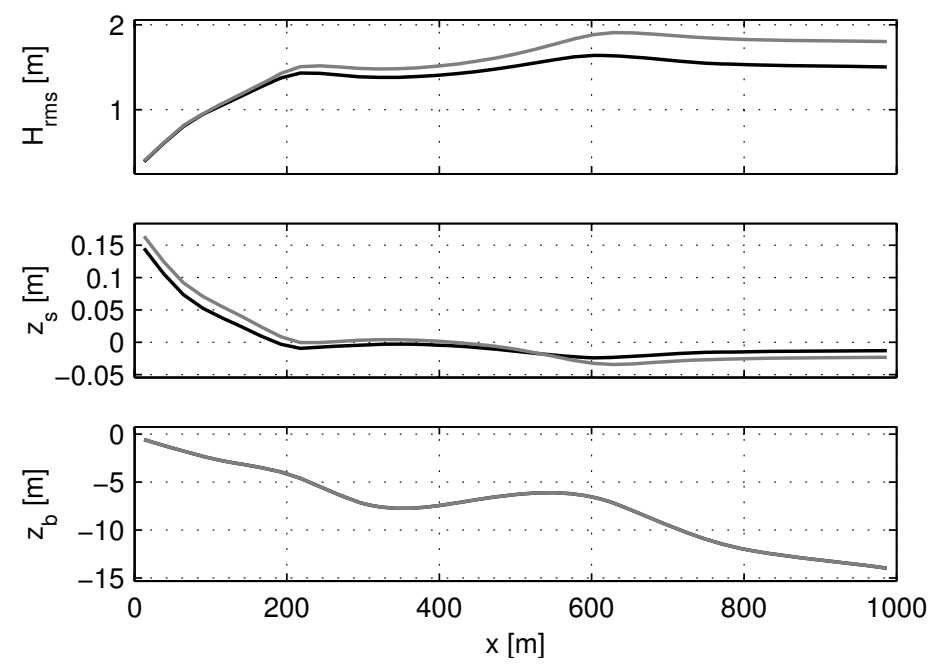

Figure 1 : États basiques. Houles frontales de hauteur $1.5 \mathrm{~m}$ (en noir) et $1.8 \mathrm{~m}$ (en gris) et de période $12 \mathrm{~s}$ au large.

Pour une condition de houle stationnaire, l'état basique est défini comme l'état d'équilibre hydrodynamique obtenu lorsque la bathymétrie initiale est uniforme longitudinalement : $z_{b}\left(x_{1}, x_{2}, t=0\right)=z_{b}^{0}\left(x_{1}\right)$. Dans ces conditions, le fond reste stable. La Figure 1 montre l'état basique obtenu pour nos deux conditions de houles frontales. Les composantes de la vitesse $v_{1}$ et $v_{2}$ ne sont pas représentées car elles sont nulles. Remarquons que, même si la dissipation d'énergie (cf. $H_{r m s}$ ) semble la même entre 0 et $200 \mathrm{~m}$ dû à la formule de dissipation de Thornton et Guza ${ }^{10}$, le setup est plus prononcé dans le cas d'une houle de $1.8 \mathrm{~m}$ (cf. $\left.z_{s}\right)$.

\subsection{Conditions de houles stationnaires}

Lorsque l'uniformité de la bathymétrie initiale est rompue, les instabilités se développent. Dans le cas d'une houle stationnaire de hauteur $1.5 \mathrm{~m}$, un état d'équilibre de barres rythmiques en croissant est obtenu, caractérisé par une longueur d'onde des barres de $650 \mathrm{~m}$ et une hauteur de $2.80 \mathrm{~m}$. La Figure 2 montre en vue 3D (seulement en une portion du domaine) l'évolution de ces barres jusqu'à l'état d'équilibre. La Figure 3 montre une vue de dessus du système de barres à l'équilibre. Remarquons la circulation associée avec un système de courants sagittaux généré dans chaque creux. La Figure 4(a) représente l'évolution temporelle de la section correspondant au sommet de la barre longitudinale où se forment les barres en croissant. Les barres rythmiques atteignent leur hauteur maximale autour de 75 jours d'évolution, ensuite elles vont décroître légèrement et interagir pour atteindre leur état d'équilibre vers 200 jours. Dans le cas d'une houle plus grosse, de $1.8 \mathrm{~m}$, comme le suggèrent les études préliminaires ${ }^{4 ; 2}$, la hauteur des barres augmente $(3.2 \mathrm{~m})$ ainsi que le taux 
d'accroissement. Comme l'indique la Figure 4(b), la hauteur maximale des barres est atteinte au bout de 45 jours. Cependant la longueur d'onde des barres à l'état final diminue, elle est de $550 \mathrm{~m}$ dans le cas (b). En effet, la variation en hauteur de vagues entre (a) et (b) est relativement faible, et, à l'état initial, les longueurs d'onde des barres sont similaires pour (a) et (b). En revanche, une houle plus grande entraîne des tensions de Reynolds plus importantes et donc moins d'interactions non linéaires. Il en résulte que, dans le cas (a) les barres ont plus tendance à s'apparier ce qui entraîne une longueur d'onde finale plus grande.
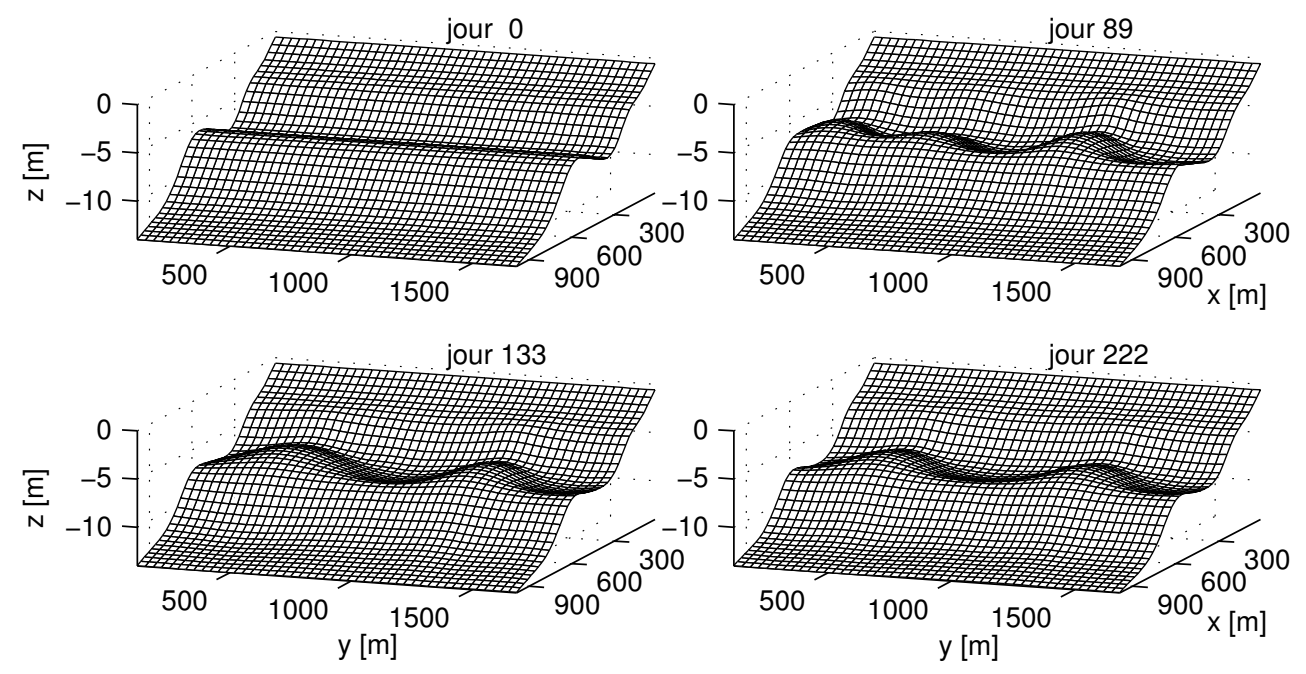

Figure 2 : Vue 3D de la topographie de la plage du Truc Vert. Houle frontale de hauteur $1.5 \mathrm{~m}$ et de période $12 \mathrm{~s}$ au large. L'axe $x$ représente la direction transversale et l'axe $y$, la direction longitudinale. L'axe $z$ correspond au niveau topographique.

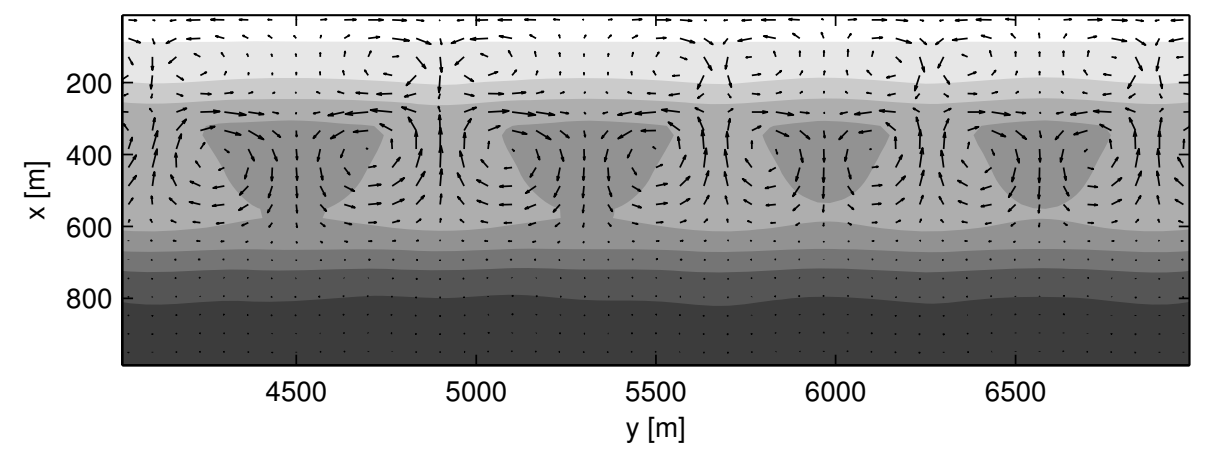

Figure 3 : État d'équilibre du système de barres en croissant (après 150 jours d'évolution). Houle frontale de hauteur $1.5 \mathrm{~m}$ et de période $12 \mathrm{~s}$ au large. Le dégradé de gris représente la bathymétrie $z_{b}$. Les zones claires (obscures) sont les zones les moins (plus) profondes. Les vecteurs représentent la vitesse $\vec{v}$.

\subsection{Conditions de houles variables}




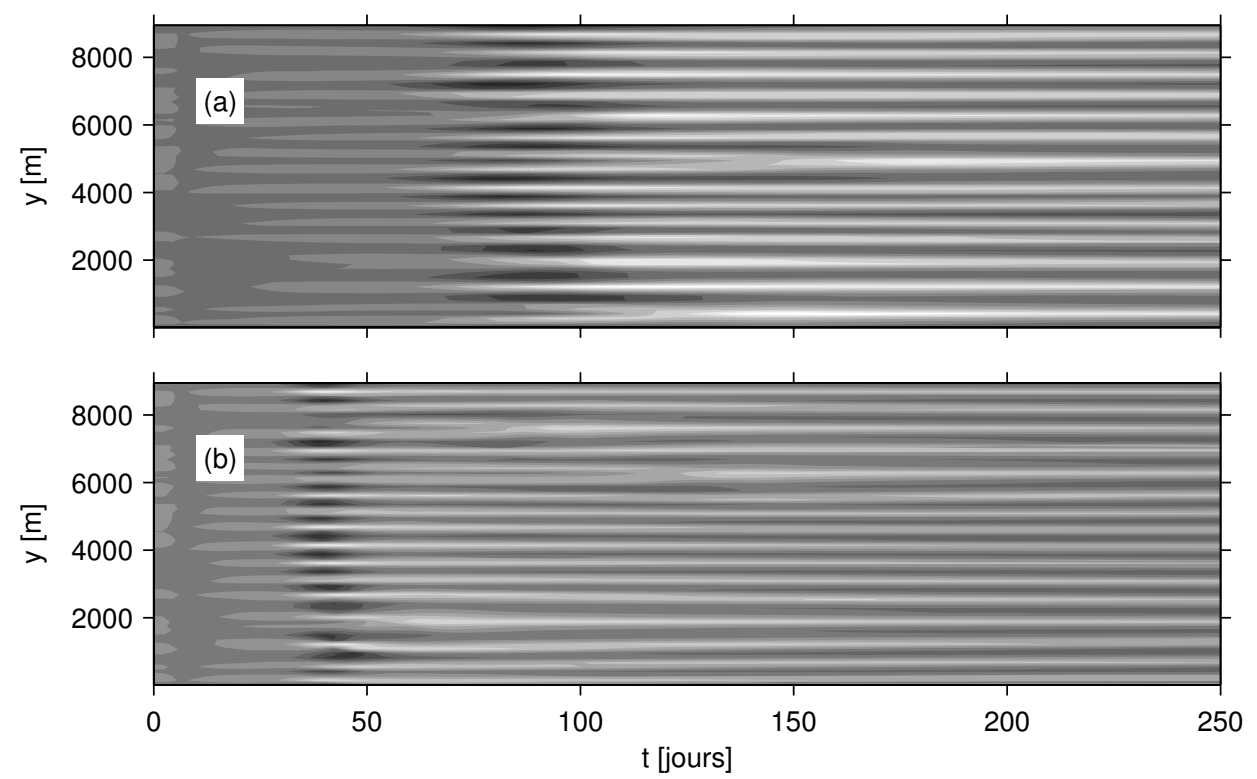

Figure 4 : Séries temporelles de la perturbation du fond le long de la section longitudinale à $x=500$ $\mathrm{m}: h(x=500, y, t)$, pour (a) $H_{r m s}=1.5 \mathrm{~m}$ et (b) $H_{r m s}=1.8 \mathrm{~m}$. Conditions de houles stationnaires.

Une houle de $1.8 \mathrm{~m}$ a été imposée sur l'état final obtenu dans le cas d'une houle de $1.5 \mathrm{~m}$ : cas $\left(\mathrm{a}_{2}\right)$. Inversement, une houle de $1.5 \mathrm{~m}$ a été imposée sur l'état final de (b) : cas $\left(b_{2}\right)$. Même si la forme des barres (asymétrie et hauteur) est retrouvée, et n'est donc pas dépendante des conditions initiales, dans aucun des cas $\left(\mathrm{a}_{2}\right) \mathrm{ni}$ $\left(b_{2}\right)$, nous ne constatons une variation de longueur d'onde du système. Ainsi, pour une hauteur de houle de $1.5 \mathrm{~m}$ ou de $1.8 \mathrm{~m}$, la longueur d'onde du système à l'état d'équilibre morphodynamique peut être de $550 \mathrm{~m}$ ou de $650 \mathrm{~m}$, selon l'état initial.

\section{Discussion et conclusion}

Les barres en croissant des plages aquitaines émergent à partir d'instabilité du système morphodynamique. Pour la première fois l'équilibre d'un tel système a été modélisé, pour une condition de houle stationnaire. Le temps au bout duquel le système a atteint son état d'équilibre est de l'ordre de 100 jours. Il est improbable que de telles conditions de houle soient observées dans la nature, cependant, nous montrons que cet état d'équilibre peut exister, les mécanismes mis en jeux sont similaires aux mécanismes de saturation décrits dans le cas des barres obliques et transversales ${ }^{6}$. Les longueurs d'ondes obtenues sont de l'ordre de $600 \mathrm{~m}$, elles correspondent avec les longueurs d'ondes observées ${ }^{3 ; 7}$.

Les études préliminaires montrant que la longueur d'onde était une fonction croissante de la taille des vagues ou plus précisément de la taille de la zone de surf sont ici étendues au régime non linéaire. Dû à des mécanismes d’interactions non 
linéaires (appariement des barres) la longueur d'onde de l'état final peut diminuer lorsque la houle grossit. De plus, l'état d'équilibre obtenu n'est pas unique, et peut dépendre des conditions initiales. Dans le cas de houle stationnaire, tous les modes étaient présents à l'instant initial du à l'ajout d'une perturbation aléatoire sur la bathymétrie initiale. Dans le cas de houle variable, la bathymétrie initiale étant l'état final d'un cas de houle stationnaire, très peu de modes sont présents, en fait, un seul prédomine largement. Les interactions non linéaires entre les modes ne sont probablement pas suffisantes pour exciter tous les modes, si bien que l'évolution est forcée à rester confinée dans son sous espace et la longueur d'onde ne varie pas.

Il sera étudié ultérieurement une plus grosse condition de houle afin (i), dans le cas d'une houle stationnaire, de vérifier si une forte augmentation de la taille de la zone de surf implique une augmentation de la longueur d'onde, (ii), dans le cas d'une houle variable, de vérifier si les barres s'apparient.

\section{Bibliographie}

1 Caballeria, M., Coco, G., Falqués, A. and Huntley, D. A. (2002). Selforganization mechanisms for the formation of nearshore crescentic and transverse sand bars. J. Fluid Mech., 465, 379-410.

2 Calvete, D., Dodd, N., Falqués, A. and van Leeuwen, S. M. (2005). Morphological development of rip channel systems : Normal and near normal wave incidence. J. Geophys. Res., 110 (C10006), doi :10.1029/2004JC002803.

3 Castelle, B. (2004). Modélisation de l'hydrodynamique sédimentaire au-dessus des barres sableuses soumises à l'action de la houle : application à la côte aquitaine. Ph.D. thesis, Université Bordeaux I, France.

4 Damgaard, J., Dodd, N., Hall, L. and Chesher, T. (2002). Morphodynamic modelling of rip channel growth. Coastal Eng., 45, 199-221.

5 Falqués, A., Coco, G. and Huntley, D. A. (2000). A mechanism for the generation of wave-driven rhythmic patterns in the surf zone. J. Geophys. Res., 105 (C10), 24071-24088.

6 Garnier, R., Calvete, D., Falqués, A. and Caballeria, M. (2006). Generation and nonlinear evolution of shore-oblique/transverse sand bars. J. Fluid Mech. In press.

7 Lafon, V., Apoluceno, D. D. M., Dupuis, H., Michel, D., Howa, H. and Froidefond, J. M. (2004). Morphodynamics of nearshore rhythmic sandbars in a mixed-energy environment (sw france) : I. mapping beach changes using visible satellite imagery. Estuarine, Coastal and Shelf Science, 61, 289-299.

8 Mei, C. C. (1989). The Applied Dynamics of Ocean Surface Waves, volume 1 of Advanced Series on Ocean Engineering. World Scientific, Singapore.

9 Soulsby, R. L. (1997). Dynamics of Marine Sands. Thomas Telford, London, U.K.

10 Thornton, B. and Guza, R. T. (1983). Transformation of wave height distribution. J. Geophys. Res., 88 (10), 5925-5938. 